\title{
Surface-induced transverse magneto-optical Kerr effect
}

\author{
A. V. Petukhov, * A. Kirilyuk, and Th. Rasing \\ Research Institute for Materials, University of Nijmegen, 6525 ED Nijmegen, The Netherlands
}

(Received 14 October 1998)

\begin{abstract}
We predict a magneto-optical Kerr rotation from a magnetized surface in the transverse geometry at normal incidence which is linear in the surface magnetization and is forbidden in the bulk. This surface-induced transverse magneto-optical Kerr effect (SITMOKE) is found to vanish at most bulk-terminated low-index faces of cubic media but is allowed at lower-symmetry planes so that SITMOKE is therefore suitable to study the magnetism of vicinal and reconstructed surfaces. The existence of SITMOKE is demonstrated by detection of an analogous strain-induced effect in a magnetic garnet film with a (210) crystallographic orientation. [S0163-1829(99)03605-X]
\end{abstract}

Symmetry breaking often has important consequences for physical properties and can lead to new effects. The surface is the most important element of translation symmetry breaking in a solid and for example may lead to reconstructions or contractions, but also to new magnetic phenomena such as enhanced or reduced magnetic moments and reorientation of the magnetic easy axis. ${ }^{1}$ In optics, the symmetry breaking at a surface leads to novel surface-induced effects like reflectance anisotropy ${ }^{2}$ and surface second harmonic generation, ${ }^{3}$ which are now widely used in surface science.

In magnetic systems the time-reversal symmetry breaking leads to the magneto-optical Kerr effect (MOKE), which has been widely explored as a relatively simple and in many respects universal tool in magnetism. ${ }^{4,5}$ Because of its sensitivity down to a submonolayer, MOKE is perfectly suitable to analyze ultrathin magnetic films. However, the effective probing depth is of the order of several hundred $\AA$ even in the case of metals, so that MOKE is not directly sensitive to the surface structure and morphology of semiinfinite magnetic media. In recent years, nonlinear magneto-optics, which is a consequence of the simultaneous breaking of space- and time-reversal symmetry, is rapidly developing into a suitable probe for the magnetism at surfaces and buried interfaces as it combines very high surface/interface sensitivity with large magneto-optical effects. ${ }^{6}$

In this paper we demonstrate that the symmetry breaking at the surface of a magnetic material also has important consequences for the linear magneto-optical response and that linear magneto-optics can be much more surface sensitive than is usually thought. A configuration is proposed in which the ordinary bulk MOKE vanishes but a surface-induced transverse magneto-optical Kerr effect (SITMOKE) appears. Similar to nonlinear optics, SITMOKE arises from an interplay between the spatial and time-reversal symmetry breaking. On the other hand, it does not require pulsed laser sources since the optical response is linear. SITMOKE is found to vanish at most bulk-terminated low-index faces of cubic crystals but is allowed at lower-symmetry planes where it shows a very distinct anisotropy. It may therefore be especially suitable to study the magnetic properties of vicinal surfaces and different surface reconstructions. Theoretical estimates indicate a detectable strength and a monolayer sensitivity for SITMOKE. The existence of this effect is experimentally demonstrated by an analogous strain-induced effect from a (210) magnetic garnet film. In that way, the effective "surface" layer thickness becomes equal to the film thickness. This enhances the signal considerably and leads to a straightforward observation of the proposed effect.

The optical response of the bulk is usually described by its dielectric constant $\overleftrightarrow{\epsilon}$, which connects the displacement $\mathbf{D}=\overleftrightarrow{\boldsymbol{\epsilon}} \cdot \mathbf{E}$ with the macroscopic optical field $\mathbf{E}$. Magnetooptical effects arise due to the dependence of $\overleftrightarrow{\epsilon}$ on the medium magnetization $\mathbf{M}$ that can be expanded up to the linear term as

$$
\overleftrightarrow{\boldsymbol{\epsilon}}(\mathbf{M})=\overleftrightarrow{\boldsymbol{\epsilon}}^{(0)}+4 \pi i \stackrel{\leftrightarrow}{f} \cdot \mathbf{M}+\cdots
$$

For cubic bulk media $\overleftrightarrow{\boldsymbol{\epsilon}}^{(0)}$ and $\overleftrightarrow{f}$ reduce to scalars $\boldsymbol{\epsilon}_{0}$ and $f_{0}$ so that the displacement can be written in the vector form

$$
\mathbf{D}(z)=\epsilon_{0} \mathbf{E}(z)+4 \pi i f_{0} \mathbf{M} \times \mathbf{E} .
$$

Therefore, within the approximation of Eq. (1), the magnetooptical interactions in a cubic bulk medium are isotropic, i.e., do not depend on the orientation of the light polarization $\mathbf{E}$ and the magnetization $\mathbf{M}$ relative to the crystallographic axes. Another important well-known consequence of Eq. (2) is that the magneto-optical Faraday and Kerr effects vanish when light propagates along a direction normal to $\mathbf{M}$ since the last term in Eq. (2) either vanishes for $\mathbf{E} \| \mathbf{M}$ or induces a nonradiative polarization along the light propagation direction for $\mathbf{E} \perp \mathbf{M}$. Thus, the transverse experimental geometry in combination with normal incidence provides the best elimination of the ordinary bulk MOKE response.

We thus consider light incident along the normal $z$ axis on a cubic semiinfinite medium magnetized along the in-plane $x$ axis: $\mathbf{M}=\hat{\mathbf{x}} M_{x}$. The reflection of light can be described by a $2 \times 2$ reflection matrix $\stackrel{\leftrightarrow}{R}$ that connects the reflected optical field $\mathbf{E}^{R}=-\overleftrightarrow{R} \cdot \mathbf{E}^{I}$ to the incident field $\mathbf{E}^{I}$ (both can be polarized along $x$ or $y$ ). If the surface-induced effects are neglected, the light reflection is described by Fresnel optics, which assumes a sharp interface. The reflection matrix $\overleftrightarrow{R}$ is then diagonal. At a real surface, however, surface-induced effects provide corrections to the Fresnel optics. The usual way to treat these effects is to split the induced polarization $\mathbf{P}(\mathbf{r}) \equiv \mathbf{P}(z)$ into its (steplike) bulk $\mathbf{P}^{B}(z)$ and surface $\mathbf{P}^{S}(z)$ parts. Since the thickness $d^{S}$ of the surface region (where $\mathbf{P}^{S}$ is nonzero) is much smaller than the light wavelength $\lambda$, the surface-induced effect is mainly determined by the total surface polarization 


$$
\mathcal{P}=\int \mathbf{P}^{S} d z=\chi_{i j}^{S}\left(E_{j}^{I}+E_{j}^{R}\right),
$$

(see Ref. 7) where $\overleftrightarrow{\chi}^{S}$ is the (integrated) surface polarizability. The surface-induced correction to the Fresnel reflection matrix can then be found as

$$
R_{i j}^{S}=\frac{8 \pi i \omega \quad \chi_{i j}^{S}}{c(n+1)^{2}},
$$

where $n$ is the refraction index of the bulk.

Although most of the coming results are valid for crystals of all five cubic symmetry classes, from here on we restrict our consideration to the $O_{h}$ class. As an example, we consider a surface with a crystallographic structure (without taking the magnetic order into account) that has one mirror symmetry plane $y^{\prime}=0$. Here $y^{\prime}$ is the crystallographic direction parallel to the surface which makes an angle $\phi$ with respect to the laboratory axis $y$. Bulk-terminated $(m n 0)$ and $(m n n)$ faces of $O_{h}$ cubic crystals have such symmetry, where $m \neq n$. The present treatment can also be easily extended for surfaces with no symmetry elements. In analogy to Eq. (1), we can write

$$
\overleftrightarrow{\chi}^{S}\left(\mathbf{M}^{S}\right)=\overleftrightarrow{X}+i \stackrel{\leftrightarrow}{F} \cdot \mathbf{M}^{S},
$$

where $\mathbf{M}^{S}$ is the magnetization of the region where $\mathbf{P}^{S} \neq 0$. Applying the surface symmetry one can find the independent elements $\quad X_{x^{\prime} x^{\prime}}=X_{0}+\Delta X, X_{y^{\prime} y^{\prime}}=X_{0}-\Delta X$, and $F_{x^{\prime} y^{\prime} x^{\prime}}$ $=-F_{y^{\prime} x^{\prime} x^{\prime}}=F_{0}$ of the relevant tensors in the twodimensional crystallographic coordinate system $\left(x^{\prime}, y^{\prime}\right)$, where $\hat{\mathbf{x}}^{\prime}=\hat{\mathbf{y}}^{\prime} \times \hat{\mathbf{z}}$. All other elements of the two-dimensional tensors $\overleftrightarrow{X}$ and $\stackrel{\leftrightarrow}{F}$ vanish. Returning back to the laboratory frame $(x, y)$, one finds

$$
\begin{aligned}
\overleftrightarrow{\chi}^{S}\left(\mathbf{M}^{S}\right)= & X_{0}\left(\begin{array}{ll}
1 & 0 \\
0 & 1
\end{array}\right)+\Delta X\left(\begin{array}{cc}
\cos 2 \phi & \sin 2 \phi \\
\sin 2 \phi & -\cos 2 \phi
\end{array}\right) \\
& +i F_{0} \cos \phi M_{x}^{S}\left(\begin{array}{cc}
0 & 1 \\
-1 & 0
\end{array}\right) .
\end{aligned}
$$

The second term on the right-hand side of Eq. (5) contains nonvanishing off-diagonal elements and is responsible for the surface-induced (nonmagnetic) reflection anisotropy. ${ }^{2}$ The last term gives rise to the surface-induced transverse magneto-optical Kerr effect (SITMOKE) that is linear in the surface magnetization $M_{x}^{S}$. Note that the SITMOKE signal should display a unique azimuthal dependence $\propto \cos \phi$ for all surfaces of cubic media that have one mirror plane. This function has onefold rotation symmetry and SITMOKE thus vanishes for the bulk-terminated low-index (100), (110), and (111) faces of $O_{h}$ cubic crystals that all possess $n$-fold rotation symmetry, where $n>1$.

The physical origin of SITMOKE can be illustrated as follows. As an example, we consider a low-symmetry (210) face of a simple-cubic crystal (Fig. 1) and assume that the main contribution to the optical response arises due to the light-induced polarization of bonds between nearestneighbor atoms (polarizable bond $\operatorname{model}^{8}$ ). The incident optical field $\mathbf{E}^{I}$ and the magnetization $\mathbf{M}$ are taken to be along the in-plane direction $\hat{\mathbf{x}}=[\overline{1} 20]$, which corresponds to $\phi$ $=0$ in Eq. (5). To couple the electron spin with its light-

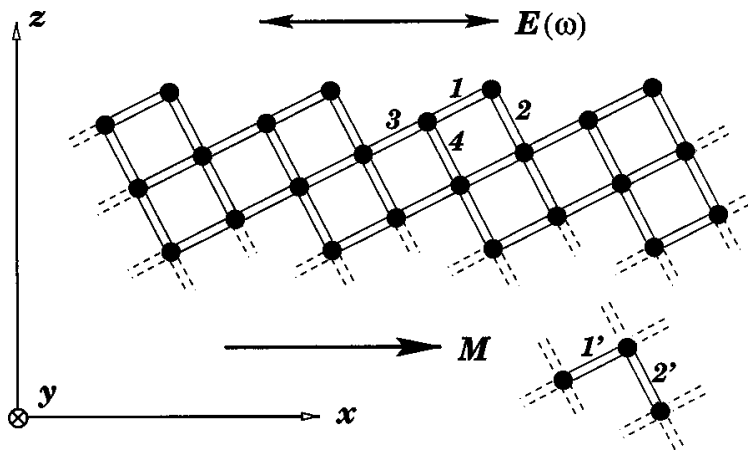

FIG. 1. A schematic side view on a (210) face of a simple-cubic crystal. Bonds $1-4$ are at the surface while $1^{\prime}, 2^{\prime}$ denote similar bonds in the bulk.

induced movement, the electron must possess a nonvanishing component of its velocity along a direction normal to $\mathbf{M}$. Since the bond is anisotropic, it is not necessarily polarized along the optical field. Thus, in a given bond $i$ a finite normal component of the bond dipole moment $d_{z}(i)$ can be (nonmagnetically) induced by the optical field. In turn, the magneto-optical interactions (spin-orbit coupling) rotate the induced moment $d_{z}(i)$, giving rise to a finite response $d_{y}(i)$ in the $y$ direction (orthogonal to the page in Fig. 1). In the bulk, however, the bond polarizations $d_{y}\left(1^{\prime}\right)$ and $d_{y}\left(2^{\prime}\right)$ induced in two networks ( $1^{\prime}$ and $2^{\prime}$ ) of bonds can be seen to cancel each other so that the macroscopic polarization of the bulk $P_{y}$ vanishes in accordance with Eq. (2).

The situation drastically changes at the surface. For example, the local optical field at the position of the surface bond 1 can be different from that at the position of the bond 2 . This asymmetry of the local field leads to a net magnetooptical response $d_{y}(1)+d_{y}(2) \neq 0$ in the $y$ direction induced in the bond pair $(1,2)$. Similarly, a nonvanishing response is found in other near-surface bond pairs: $(3,4)$, etc. Therefore, the off-diagonal element $\chi_{y x}^{S}\left(\mathbf{M}^{S}\right)$ is induced at the surface due to magneto-optical interactions. Going from the surface further into the bulk the local fields approach their bulk values and the sum $d_{y}(i)+d_{y}(i+1)$ vanishes at distances of the order of several lattice constants. Our preliminary estimates ${ }^{9}$ show that the local field effect alone can yield a SITMOKE response that is comparable to the strength of ordinary polar MOKE from a monolayer of the same magnetic material.

The local field effect is not a unique source of SITMOKE. For example, the electronic states forming the surface bonds are not the same as those forming the bulk bonds because of their different environment. The (nonmagnetic) polarizability of the surface bond $i$ can then differ from that of the bond $i+1$, leading to an additional asymmetry of the $y$ components of the bond dipole moments $d_{y}(i)$ and $-d_{y}(i+1)$. This effect can be significantly enhanced if a surface relaxation takes place (not shown in Fig. 1). For example, a slight displacement of the step atom from its bulk-terminated position results in different lengths of the bonds 1 and 2 and, thus, in a stronger difference between the bond polarizabilities. The strength of the magnetic interactions in nearsurface bonds can also be altered, for instance because of the enhanced or reduced magnetic moments $|\boldsymbol{\mu}(i)|$ associated with the $i$ th bond at the surface. Moreover, the direction of 


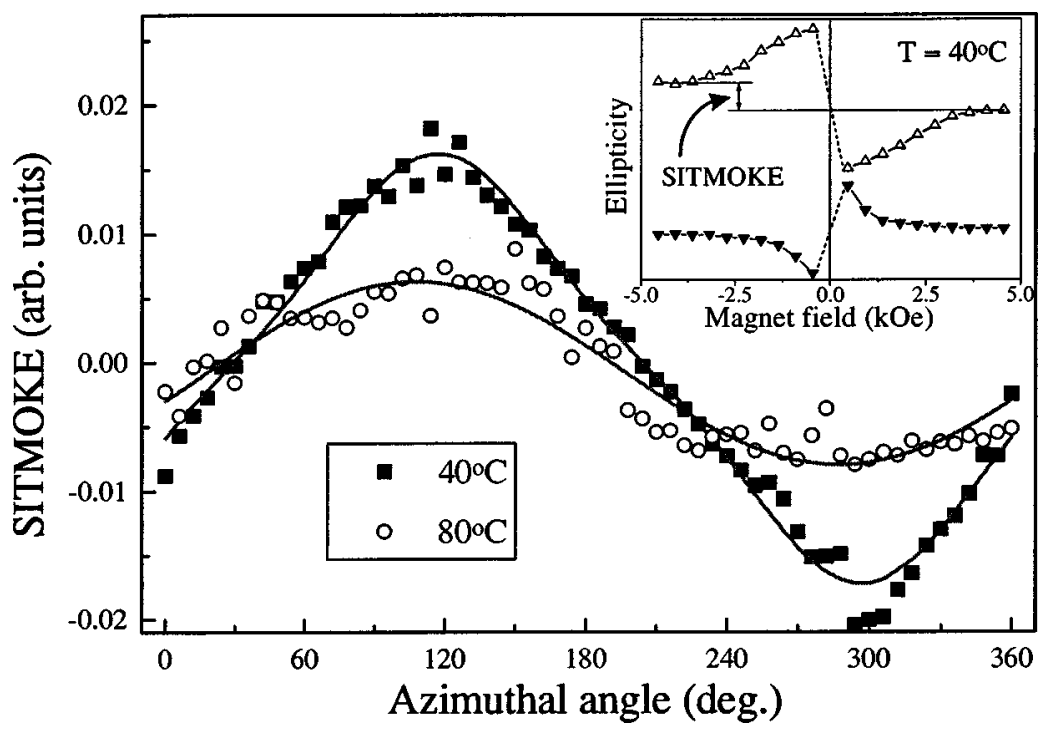

FIG. 2. Azimuthal dependence of the strain-induced effect, analogous to SITMOKE, $\mathcal{I}(\phi)$, measured at temperatures $T=40^{\circ}$ (squares) and $T=80^{\circ}$ (circles). The lines show the fit of the data as described in the text. Hysteresis loops $\delta I_{\Omega}^{T}\left(H_{x}\right)$ at $T=40^{\circ}$ for two particular azimuthal angles $\phi=300^{\circ}$ (open triangles) and $180^{\circ}$ (closed triangles) are shown in the inset.

$\boldsymbol{\mu}(i)$ can deviate from the direction of $\boldsymbol{\mu}\left(i^{\prime}\right)$ for a similar bond in the bulk. In addition, for the structure shown in Fig. 1 there are three dangling bonds per surface unit cell (not shown), which can contribute to SITMOKE especially when the light resonantly excites surface states associated with the dangling bonds. All the effects mentioned above are only possible in a narrow vicinity of the surface and, therefore, SITMOKE should be sensitive to the surface magnetization.

It is worth noting that the assumption of the anisotropy of the bond polarizabilities, which is used above, is not necessary in order to obtain a finite SITMOKE response. Numerical estimates of the optical response of spherically symmetric atoms in the structure displayed in Fig. 1 show that the asymmetric arrangement of nearest atoms causes the local field at the position of a surface atom to possess a finite (nonmagnetically induced) normal $z$ component. In turn, this field induces a response that is coupled to the magnetization and leads to SITMOKE with an intensity of order 10-20\% of the intensity of the polar MOKE from a monolayer-thick magnetic film. ${ }^{9}$

To explore the surface sensitivity of SITMOKE one needs a sensitive detection system, similar to that used in Ref. 5 and a proper UHV system suitable for normal-incidence MOKE measurements. However, the existence of such a transverse effect can be more easily demonstrated by a much simpler experiment. As a "model" of the magnetized surface we take a (210)-oriented magnetic garnet film, epitaxially grown on a nonmagnetic garnet substrate. It was found $^{10,11}$ that the structure of such a film is slightly distorted by the growth-induced strain along the film normal, while the in-plane symmetry coincides with that of the substrate surface. Due to this distortion, a strain-induced response, analogous to SITMOKE, comes from the whole magnetic film (with a thickness of $10 \mu \mathrm{m}$ ), if measured in transmission. Note that for an actual SITMOKE experiment on a real surface the Kerr (reflection) configuration should provide better elimination of the bulk contributions and it therefore should be more suitable than the Faraday geometry used here.

The sample was placed between the poles of a magnet. The incoming light with $\lambda=632.8 \mathrm{~nm}$ from a He-Ne laser was polarized at $45^{\circ}$ with respect to the direction $x$ of the magnetic field and passed a photoelastic modulator that provided a time-dependent phase shift $\delta(t)=(\pi / 2) \sin (\Omega t)$ between the $x$ and $y$ components. The light beam was then transmitted through the sample and the modulation amplitude $\delta I_{\Omega}^{T}\left(H_{x}\right)$ at frequency $\Omega$ of the $x$-polarized transmitted light intensity $I_{x}^{T}$ (that probes the ellipticity) was recorded using a lock-in detection technique. ${ }^{12}$ The "SITMOKE', signal is defined as

$$
\mathcal{I}=\delta I_{\Omega}^{T}\left(H_{x}^{\mathrm{sat}}\right)-\delta I_{\Omega}^{T}\left(-H_{x}^{\mathrm{sat}}\right),
$$

where $H_{x}^{\text {sat }}$ is the saturation field along the $x$ direction. The experimental data (Fig. 2) closely follow the $\cos \phi$ azimuthal dependence at temperatures $T \gtrsim 60^{\circ} \mathrm{C}$ while at lower $T$ the data can be better fitted by the function $A \cos \phi+B \cos ^{3} \phi$, where $A$ and $B$ are temperature-dependent constants.

To understand the origin of the additional contribution to the measured magneto-optical rotation one has to include effects that are neglected above but can be present in unstrained cubic bulk media. In particular, further terms in the expansion $\boldsymbol{\epsilon}(\mathbf{M})$, Eq. (1), could become important yielding a bulk contribution to the nonreciprocal Kerr effect in the transverse configuration that is of third order in $M_{x} \cdot{ }^{13,14}$ The azimuthal dependence of this bulk contribution is however distinctly different from the $\cos \phi$ dependence that is characteristic for SITMOKE. For instance, for the (210) surface of cubic crystals the bulk contributions can be shown ${ }^{14}$ to yield a $\Phi_{(210)}^{\text {bulk }}(\phi)=\cos ^{3} \phi$ pattern. Also, the fourth-order cubic anisotropy can lead to a deviation of the direction of the bulk magnetization $\mathbf{M}$ from the direction of the external field $\mathbf{H}$ and thus to an ordinary MOKE response. This contribution 


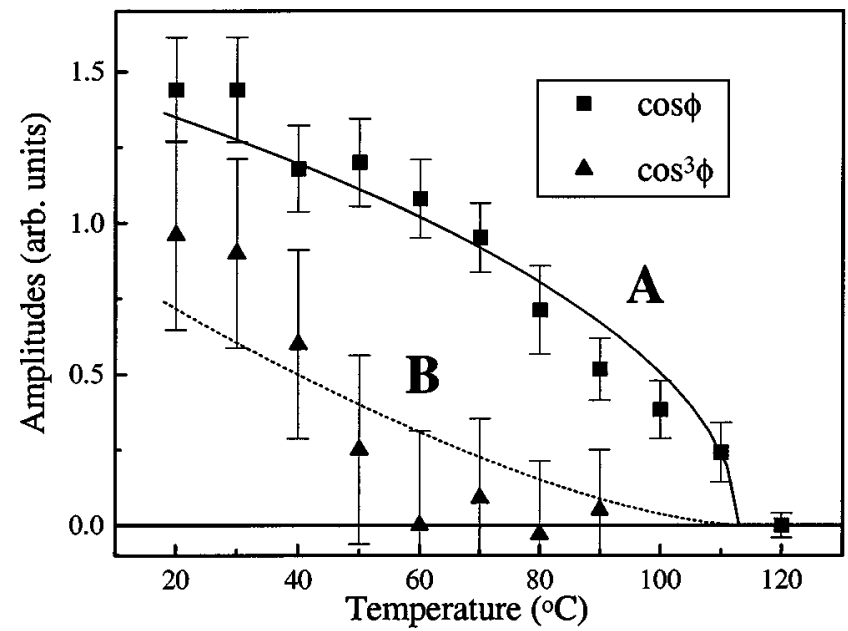

FIG. 3. Temperature dependence of the amplitudes $A(T)$ (squares) and $B(T)$ (triangles). The lines show the $\left(T-T_{c}\right)^{1 / 2}$ and $\left(T-T_{c}\right)^{3 / 2}$ dependencies, where $T_{c}=112^{\circ}$ is the Curie temperature of the magnetic film.

can be shown to be proportional to $M^{4} / H$ at large $H$ and to follow for the (210) face the same $\Phi_{(210)}^{\text {bulk }}=\cos ^{3} \phi$ azimuthal variation as the higher-order contribution to the $\epsilon(\mathbf{M})$ dependence discussed above. The increase of the light ellipticity at smaller $H$ (that can be seen in the inset in Fig. 2) is related to the latter effect so that the SITMOKE signal must be defined as shown in Eq. (6).

Thus, all the bulk contributions to the transverse magnetooptical effect should display the same azimuthal variation $\Phi^{\text {bulk }}(\phi)$, which is distinctly different from the $\cos \phi$ dependence of the surface-induced response. In addition, the surface-induced and bulk effects should display distinctly different temperature dependences because of their different dependence on M. Figure 3 shows the temperature dependence of the amplitudes $A(T)$ and $B(T)$ obtained from the fits of the data $\mathcal{I}(\phi, T)$ to the function $A(T) \cos \phi$ $+B(T) \cos ^{3} \phi$. The measured $A(T)$ dependence closely follows a $\left(T-T_{c}\right)^{1 / 2}$ function, which is as expected since the strain-induced effect, analogous to SITMOKE, is linear in the magnetization. On the other hand, the amplitude $B(T)$ of the higher-order bulk contributions should decay as $M^{n}(T)$ with $n \geqslant 3$ when the temperature $T$ is increased. The experimental result for the $B(T)$ dependence is in agreement with this prediction (Fig. 3).

In summary, we propose a new linear magneto-optical configuration that can be used to observe a surface-induced transverse magneto-optical Kerr effect (SITMOKE). Possible physical mechanisms of SITMOKE are discussed. We argue that the sources contributing to this magneto-optical response are localized in a narrow near-surface region so that SITMOKE should be sensitive to the surface magnetization and can be applied to probe the magnetism of low-symmetry surfaces and interfaces such as vicinal or reconstructed surfaces. A theretical estimate ${ }^{9}$ shows the SITMOKE effect to be of a comparable strength as that of the polar Kerr effect of a magnetic monolayer, nowadays easily detectable. ${ }^{5}$ The existence of the proposed effect is demonstrated in a model experiment using a strained (210) garnet film that is characterized by the symmetry of the bulk-terminated (210) face of a cubic crystal. The higher-order bulk contributions could be distinguished from the SITMOKE signal by their different azimuthal and temperature dependences. It should be stressed that using a bulk garnet film for demonstration does not alter anything to the conclusion that SITMOKE is an intrinsically surface sensitive magneto-optical probe.

Valuable discussions with Roman V. Pisarev, John E. Inglesfield, and Annalisa Fasolino are gratefully acknowledged. The present work was partially supported by the HCM fellowships ERBCHBICT941761, ERBCHRXCT940563, Technologiestichting STW, and TMR network "NOMOKE."
*On leave from the Institute of Crystallography, 117333 Moscow, Russia.

${ }^{1}$ U. Gradmann, J. Magn. Magn. Mater. 100, 481 (1991); L. Falicov and D. T. Pierce, J. Mater. Res. 5, 1299 (1990), and references therein.

${ }^{2}$ K. Hingerl, D. E. Aspnes, I. Kamiya, and L. T. Florez, Appl. Phys. Lett. 63, 885 (1993); Y. Borensztein, W. L. Mochan, J. Tarriba, R. G. Barrera, and A. Tadjeddine, Phys. Rev. Lett. 71, 2334 (1993); J. McGilp, Prog. Surf. Sci. 49, 1 (1995).

${ }^{3}$ Y. R. Shen, Solid State Commun. 102, 221 (1997).

${ }^{4}$ S. D. Bader and J. L. Erkine, in Ultrathin Magnetic Structures, edited by B. Heinrich and J. A. C. Bland (Springer-Verlag, 1994); S. M. Jordan and J. S. S. Whiting, Rev. Sci. Instrum. 67, 4286 (1996).

${ }^{5}$ S. D. Bader, J. Magn. Magn. Mater. 100, 440 (1991).

${ }^{6}$ R.-P. Pan, H. D. Wei, and Y. R. Shen, Phys. Rev. B 39, 1229 (1989); W. Hübner and K. H. Bennemann, ibid. 52, 13411 (1995); B. Koopmans, M. G. Koerkamp, Th. Rasing, and H. v.d. Berg, Phys. Rev. Lett. 74, 3692 (1995); M. Straub, R. Vollmer, and J. Kirschner, ibid. 77, 743 (1996); T. M. Crawford, C. T.
Rogers, T. J. Silva, and Y. K. Kim, Appl. Phys. Lett. 68, 1573 (1996).

${ }^{7}$ J. E. Sipe, Surf. Sci. 84, 75 (1979).

${ }^{8}$ C. D. Hogan and C. H. Patterson, Phys. Rev. B 57, 14843 (1998); B. S. Mendoza and W. L. Mochán, ibid. 55, 2489 (1997); P. L. de Boeij, C. M. J. Wijers and E. Zoethout, J. Vac. Sci. Technol. B 14, 3080 (1996).

${ }^{9}$ A. V. Petukhov et al. (unpublished).

${ }^{10}$ R. V. Pisarev, B. B. Krichevtsov, V. N. Gridnev, V. P. Klin, D. Frohlich, and Ch. Pahlke-Lerch, J. Phys.: Condens. Matter 5, 8621 (1993).

${ }^{11}$ V. V. Pavlov, R. V. Pisarev, A. Kirilyuk, and Th. Rasing, Phys. Rev. Lett. 78, 2004 (1997).

${ }^{12}$ K. Sato, in Magnetic Multilayers, edited by L. H. Bennett and R.E. Watson (World Scientific, Singapore, 1994), pp. 277-297.

${ }^{13}$ V. N. Gridnev, B. B. Krichevtsov, V. V. Pavlov, and R. V. Pisarev, Pis'ma Zh. Éksp. Teor. Fiz. 65, 65 (1997) [JETP Lett. 65, 68 (1997)].

${ }^{14}$ A. V. Petukhov, Th. Rasing, T. Katayama, N. Nakajima, and Y. Suzuki, J. Appl. Phys. 83, 6742 (1998). 